http://dx.doi.org/10.21611/qirt.1994.040

\title{
Reconstruction of thermal defects from photothermal images
}

\author{
by LAN T.T.N., HAUPT K., SEIDEL U. and WALTHER H.G.
}

Institute of Optics and Quantum Electronics,

Max-Wien-Platz 1, 07743 JENA, Germany

\section{Abstract}

The paper reports on 2 methods to get depth dependend information about thermal properties from photothermal measurements. First, an inversion technique is suggested to calculate the depth profile of either $k$ or ce. Second, a deconvolution technique making use of the photothermal point spread function is presented to estimate depth, size and strength of buried localized thermal defects.

\section{Nomenclature}

A signal amplitude

$A^{\prime} \quad$ amplitude ratio

c specific heat

D thermal defect distribution

f

$F$

G

function (PSF)

$\mathrm{H}$ absorbed heat density

k thermal conductivity

$\mathrm{K}$ photothermal contrast

$N$ number of measurements

$P^{\prime} \quad$ phase difference

$P \quad$ signal phase

S complex photothermal signal

$W$ window function

$x, y \quad l$ lateral coordinates

$z$ depth coordinate

\section{Subscripts}

b bulk

calc calculated

$i, m, n$, running indexes

meas measured

ref reference

o homogeneous
Greek symbols

@ density

$\sigma$ thermal wave number

$\Delta z$ defect thickness

\section{Introduction}

Time resolved thermography enables us to see behind the surface of optically opaque samples. Thermal waves generated by periodical sample heating provide information about hidden defects and inhomogeneities. Because of their dispersive propagation velocity $v(f) \doteq$ $2(\pi \mathrm{kf} / \mathrm{oc})^{1 / 2}$ they show a characteristic phase lag to excitationdepending both on the distance between irregularity and surface and on modulation frequency.

The advantage of surface detected thermal waves was exploited by us

- to reconstruct the depth profile of thermal properties ( as it is of interest in case of dopant profiles, hardening layers,...) 
http://dx.doi.org/10.21611/qirt.1994.040

- to reconstruct depth, shape and strength of localized perturbations of thermal properties within an otherwise homogeneous material.

\section{Reconstruction of thermal conductivity depth profile}

\subsection{Mathematical approach of inversion}

The inhomogeneous heat diffusion equation can be solved analytically in the case of linearly inhomogeneous $\mathrm{k}$ or $\mathrm{ec}$ [1]. So, for example, it is possible to calculate analytically the surface temperature of a surface absorbing sample with a k-profile shaped like a polygonal curve.

Knowing this result it is possible to invert the measured surface temperature by an inversion procedure and to obtain the polygonal k-profile. The approach is the following:

The surface temperature depending on modulation frequency is measured by an appropriate photothermal technique ( eg. radiometrically), yielding the complex photothermal signal

$$
S(0, t)=A(f) e^{i P(f)+i 2 n t t}
$$

It is assumed that i) the property $\mathrm{gc}$ is constant inside the whole sample and ii) the bulk thermal conductivity is known.

For further considerations we have to take into account the measured curves of the amplitude ratio $A^{\prime}=A / A_{b}$ and the phase difference $P^{\prime}=P-P_{b}$. The subscript $b$ denotes the pure bulk material used as a reference. To reconstruct approximately the k-profil of the sample under test by a polygonal curve we suppose the subsurface region being subdivided into sublayers of equal thickness $\Delta z . z_{i}$ are the interface depths.

Approximately we obtain the surface value $k_{0}=k\left(z_{0}\right)$ by the relation

$$
k_{0}=k_{b} /\left(A^{\prime}\left(f_{0}\right)\right)^{2}
$$

where the measured amplitude ratio is taken at the maximum adjustable frequency $f_{0}$.

Starting from $k_{0}$ we approximate $k$ at each sublayer interface $z_{m}$. Within each sublayer $k$ varies linearly. If we know all the $k$ values from $z_{0}$ to $z_{m}$, how can we get $k\left(z_{m+1}\right)=$ $k\left(z_{m}+\Delta z\right)=k_{m+1}$ ?

Decreasing the modulation frequency from $f_{m}$ to a lower value $f_{m+1}$ the thermal waves provide additional information about the sublayer between $z_{m}$ and $z_{m+1} \cdot f_{m+1}$ is obtained from the relation

$$
. z_{m+1}-z_{m}=d z=\left[\left(k_{b}-k_{m}\right) / 2 \pi \varrho c\right]^{1 / 2}\left[\left(1 / f_{m}\right)^{1 / 2}{ }_{-}\left(1 / f_{m+1}\right)^{1 / 2}\right]
$$

The next value $k_{m+1}$ can be obtained by a least square fit comparing. either the measured amplitude ratios or phase differences with the corresponding calculated ones

$$
\left(\sum\left[A_{\text {meas }}^{\prime}\left(f_{i}\right)-A_{\text {calc }}^{\prime}\left(f_{i}\right)\right]^{2} /\left[N\left(f_{m+1}\right)-1\right]\right)^{1 / 2}=\operatorname{Min}
$$

$N\left(f_{m+1}\right)$ is the number of all the measurement frequencies $f_{i}$ in the range from $f_{0}$ to $f_{m+1}$. Equ. (4) has to be minimized only with respect to $k_{m+1}, B y$ this approach we "build-up". the kprofile from the surface to the depth using the data from very high modulation frequencies to lower ones. The applicated least square technique allows for a good reconstruction also in the case of unproperly chosen surface conductivity.

A similar inversion procedure can be carried out in the case of a sample with gradually linearly inhomogeneous $\mathrm{Q} \epsilon$ but constant $k$.

\subsection{Numerical inversion results from synthesized $k$ profiles}


http://dx.doi.org/10.21611/qirt.1994.040

We tested the ability of the inversion technique by presuming a polygonal k-profile. The photothermal signal which is proportional to the surface temperature was calculated using the analytical solution of the 1-dimensional heat diffusion equation. Then the frequency dependent $A^{\prime}$ and $P^{\prime}$ curves were estimated.

Summarizing all of our numerical inversion results we may conclude that inverting the ph ise data gives a better $k$-profile reconstruction than inverting the amplitude data. This cr -responds to the well known behavior of the 1-dimensional thermal waves to produce higher contrast in phase than in amplitude from deeply buried interfaces.

The performance of the proposed inversion technique is demonstrated in Fig. 1. Here a nonmonotonic k-profil has to be reconstructed from noisy phase measurements. The random phase error was assumed to be $0.2^{\circ}$. Subdividing the subsurface region into 25 sublayers gives a good approximation of the actual profile. The approximation can be improved by increasing the number of sublayers, decreasing their thicknesses and higher grading the slope steps in $\mathrm{k}$.

Moreover, the proposed technique is rather insensitive to noise of the measurement, as it is shown in the next figure. In this example the phase noise was assumed to be $1^{\circ}$, a rather high but realistic value ( amplitude noise needed for the estimation of $k_{\circ}$ was $10 \%$ in this case). Nevertheless, the k-profile could be reconstructed succesfully from noisy data. In Fig. 2 several reconstructed profiles are shown corresponding to different "experimental" realizations of the same synthesized profile. The individual reconstructions are close together demonstrating the good convergence of the method.

\section{Reconstruction of localized thermal defects}

\subsection{Deconvolution using the photothermal point spread function}

Due to the diffusive nature of thermal waves and their mutual interference the images of buried objects are blurred. Resemblance between image and object fades out with increasing depth of the object.

At photothermal imaging there arises the necessity to reconstruct buried defects from photothermal images. We present a first attempt to make use of the photothermal point spread function (as defined in [2]) for object reconstruction. The method is based on the property of the photothermal contrast image which can be expressed mathematically as a convolution integral of the point spread function $G$ and a distribution function $D$ of thermal inhomogeneities. The measurable photothermal contrast as defined in [3] can be written as

$$
K(x, y)=\Delta z \iint G\left(x-x^{\prime}, y-y^{\prime}, z_{0}\right) D\left(x^{\prime}, y^{\prime}, z_{0}\right) d x^{\prime} d y^{\prime}
$$

assuming a defect of small thickness $\Delta z$, buried at depth $z_{0} . D(x, y, z)$ describes the distribution of the normalized parameter deviations

$$
D_{k}=\left(k(x, y, z)-k_{0}\right) / k_{0}, \quad, D_{\rho c}=\left(\varrho c(x, y, z)-\varrho c_{0}\right) / \varrho c_{0}
$$

We have to keep in mind that $G$ depends on both the defect depth and the type of thermal inhomogeneity (thermal conductivity and/or thermal density).

From the measured complex valued contrast $\mathrm{K}=\mathrm{K}_{\mathrm{A}}+\mathrm{i} \mathrm{K}_{\mathrm{p}}$ we can obtain the defect distribution by appropriate deconvolution. By Fourier transforming all mathematical expressions $\mathrm{FT}\{\mathrm{D}\}$ can be obtained as ratio $\mathrm{FT}\{\mathrm{K}\} / \mathrm{FT}\{\mathrm{G}\}$. But, in the experiments, there are at least 2 problems:

1) $K$ results from noisy measurements. 
2) Both the defect depth $z_{0}$ and defect type usually are unknown and have to be estima ted by the reconstruction procedure.

To overcome the noise difficulties we have to multiply $\mathrm{FT}\{\mathrm{K}\}$ by an appropriately chosen window spektrum FT $\{\mathrm{W}\}$

$$
D=1 / \Delta z F T^{-1}\{F T\{K\} F T\{W\} / F T\{G\}\}
$$

Obviously, we only obtain the true defect distribution $D\left(x, y, z_{0}\right)$ as the inverse Fourier transfor$m$ if we deconvolute it with the correct PSF (taken at the correct depth $z=z_{0}$ and knowing the thermal type of defect). Of course, these values are unknown, and we have to determine the correct PSF $G$ from an independent criterion. For simplicity, we restrict our efforts to the search of the unknown defect depth $z_{0}$ and deconvolute the "measured" contrast $K(x, y$,$) with$ a number of PSF's taken at guessed depths z. Complex valued defect distributions are obtained whose imaginary content $F$ is quantified by

$$
F=\left[\sum \operatorname{Im}\{D(x, y, z)\}^{2}\right]^{1 / 2}
$$

Because $D\left(x, y, z_{0}\right)$ has to be a real valued function we will find the correct depth $z=z_{0}$ if $F=0$ or, in the presence of noise, if $F$ has a minimum among the various depths investigated. Numerically, this means to find the global minimum of the function $F=F(z)$. Fig. 3 shows the typical behavior of $F(z)$ in the surrounding of $z_{0}$ with its global minimum.

\subsection{Reconstruction of a buried strip defect}

We designed a sample which matches as much as possible the assumptions made by the inversion model:

- small thermal difference between defect and surrounding medium,

- sample is a surface absorber (metal)

- the defect thickness is small compared to both the defect width and the thermal diffusion length.

Also, the assumption of signal being proportional to the averaged surface temperature was satisfied by wide aperture radiometric detection (active area of the detector: $1 \mathrm{~mm}^{2}$ ). Due to experimental conditions, the assumption of pointlike excitation was not fulfilled. This could result in a further blurring of the image.

The sample was made out of a soldering tin-alloy (96\%Sn, $4 \% \mathrm{Ag}$ ). The defect strip was embedded $200 \mu \mathrm{m}$ below the surface, $5 \mathrm{~mm}$ from each side of the sample to avoid reflection of the thermal waves even at low frequencies. The strip consists of stainless steel, it is 50 $\mu \mathrm{m}$ thick and $500 \mu \mathrm{m}$ wide. The raw data were recorded within the frequency range from 15 to $1400 \mathrm{~Hz}$, selected scans are given in Fig.4. From these data amplitude and phase contrast have been calculated followed by smoothing with window functions adapted for the problem.

After calculating Equs. (7) and (8) we found the defect being situated at depth $z_{0}=270 \mu \mathrm{m}$ (actual: $225 \mu \mathrm{m}$ ) and having the "contour" as shown in Fig.5. A strip-like shape of width about $700 \mu \mathrm{m}$ (actual: $500 \mu \mathrm{m}$ ) has been found. This broadening is caused, among other reasons, by the finite width of the heating laser beam. From the magnitude of the center contrast, especially from its frequency dependence, we could estimate the magnitude of the deviations in $\mathrm{k}$ and $\varrho \mathrm{c}$ to be $\Delta \mathrm{k} / \mathrm{k}_{0}=0.7$ (actual: 0.7 ) and $\Delta \varrho \mathrm{\rho c} / \mathrm{ec}_{0}=-1.25$ (actual: -0.4 ). The good correspondence between actual and calculated $k$-distortion has to be pointed out. The larger difference of the ec-distortion may be due to its generally smaller contribution to the photothermal signal. 


\section{REFERENCES}

[1] FRIEDRICH (K.), SEIDEL (U), WALTHER (H.G.), KARPEN (W.) and BUSSE (G.) "Proposal for Photothermal Characterization of Boundaries Between Layer and Substrate", Res. Non destr. Eval. 5, 1993, 31-39

[2] FRIEDRICH (K.), HAUPT (K.), SEIDEL (U), and WALTHER (H.G.) - "Definition, Resolution and Contrast in Photothermal Imaging", J.Appl.Phys. 72, 1992, 3759-3764

[3] SEIDEL (U), HAUPT (K.), WALTHER (H.G.), BUTR (J.) and BEIN (B.K.) - "Analysis of the Detectability of Buried Inhomogeneities by Means of Photothermal Microscopy", J.Apply.Phys. 75, 1994, 4396-4401

Phase Difference [degrees]

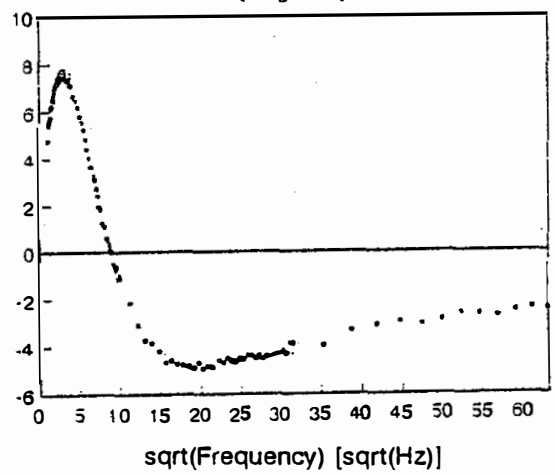

Thermal conductivity $\mathrm{k}[\mathrm{W} / \mathrm{Kcm}]$

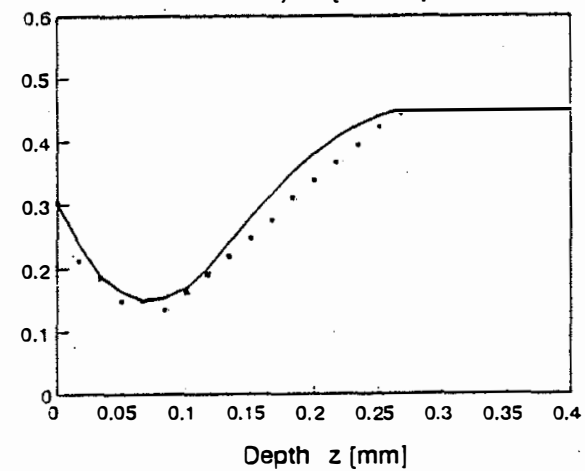

Phase difference [degrees]

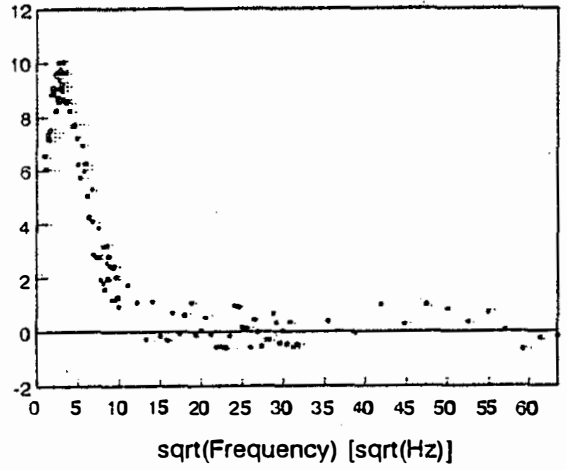

Thermal conductivity $k[\mathrm{~W} / \mathrm{Kcm}]$

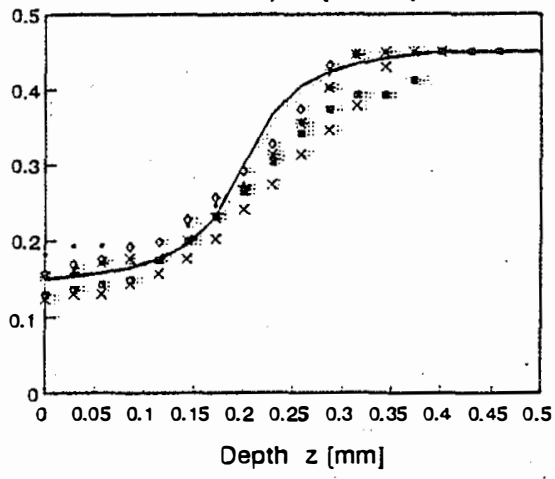

Fig.2: Reconstruction of a k-profile from noisy phase measurements (phase error $1^{\circ}$ ) 
http://dx.doi.org/10.21611/qirt.1994.040

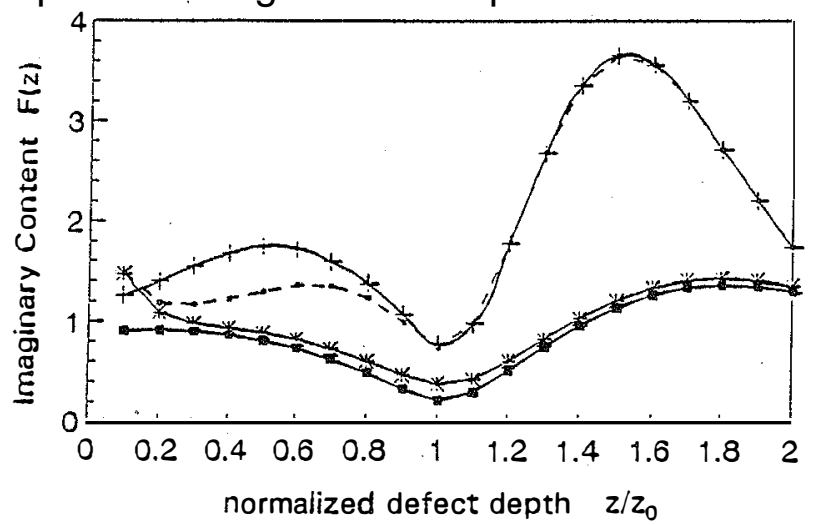

Fig.3: Dependence of the imaginary contrast of $D$ on guessed defect depths $z$ (true depth $z_{0}$ )
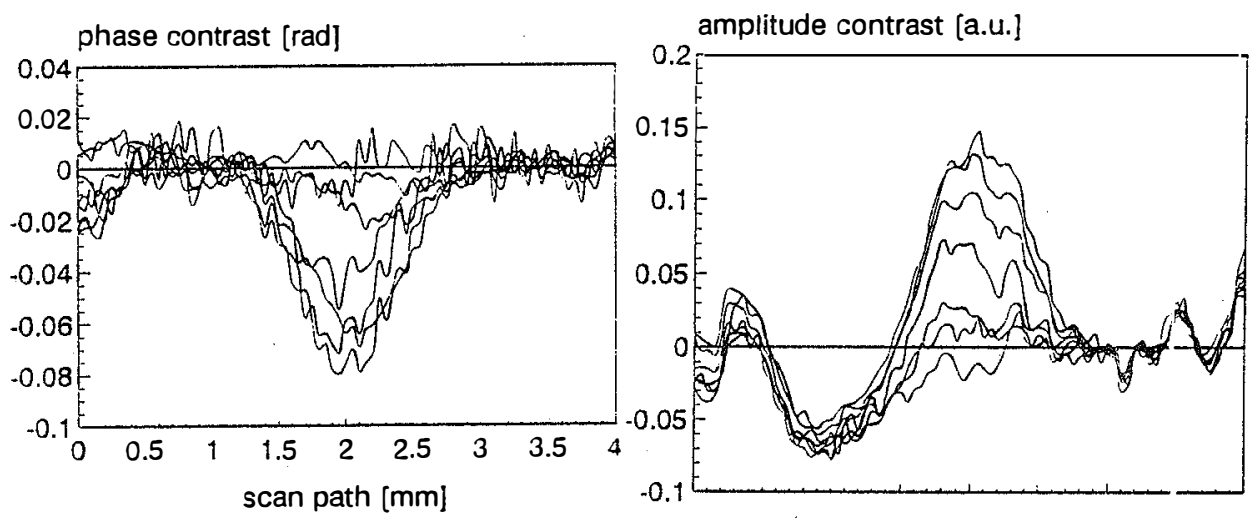

Fig.4: Radiometric scans across the buried strip at different frequencies

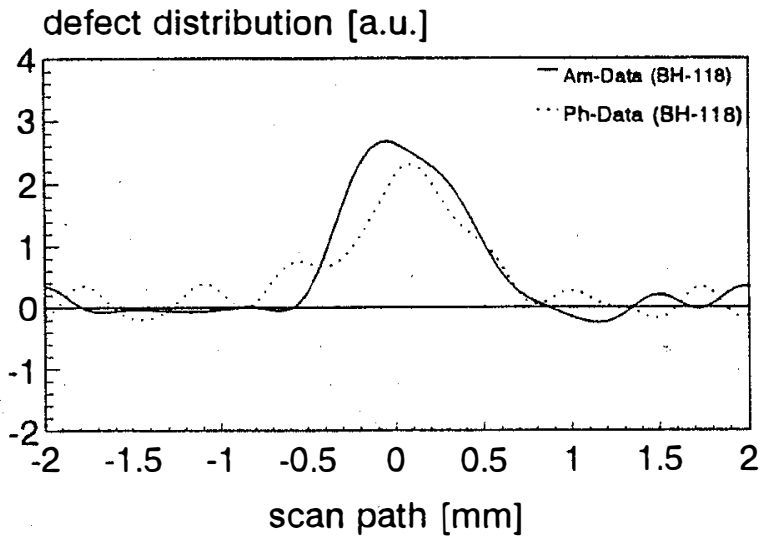

Fig.5: Reconstructed contour of the buried metal strip 